\title{
4 Bewerten im Kontext geselliger Interaktion
}

Mit Blick auf die in der vorliegenden Arbeit untersuchten Gespräche spielt neben dem in Kapitel 3.1 bereits betrachteten Kontext der Kunstkommunikation ebenso der gesellige Kontext in der Interaktion der Beteiligten eine wichtige Rolle. Dass der Theaterbesuch für die Zuschauer auch ein geselliges Ereignis darstellt, wird unter anderem an den in den Gesprächen in unterschiedlichem Umfang enthaltenen Anteilen von Small Talk und phatischer Kommunikation deutlich (vgl. Linz/ Hrncal/Schlinkmann 2016: 529; Gerwinski/Habscheid/Linz 2018 sowie Linz 2018). Im Fluchtpunkt dieses Kapitels stehen deshalb die Fragen, welche Funktionen phatische Kommun(ikat)ion, Small Talk und homileïscher Diskurs im Rahmen geselliger Kommunikation haben, und wie der Kontext der geselligen Interaktion die Gesprächsteilnehmer dabei unterstützen kann, im prestigeträchtigen Kommunikationsraum der Kunst (vgl. Müller/Kluwe 2012: 5) mit potenziell heiklen Positionierungen und Face-Bedrohungen umzugehen, die - wie in Kapitel 2 dargelegt - mit Bewertungen verbunden sind.

\subsection{Phatische Kommunion, Small Talk und homileïscher Diskurs}

Mit dem Terminus ,phatic communion' (,speech in social intercourse“) referiert Malinowski (1923/1936: 296; vgl. auch Senft 2009: 226) auf nicht-zielgerichtete Interaktion, in der keine Informationen eingeführt werden und keine Bedeutung übermittelt wird, sondern die dazu dient, eine Beziehung zwischen den Interagierenden zu schaffen: „,phatic communion' [. . .] serves to establish bonds of personal union between people brought together by the mere need of companionship and does not serve any purpose of communicating ideas" (Malinowski 1923/1936: 316). ${ }^{38}$ In phatischer Kommunion erfüllen Äußerungen somit in erster Linie eine soziale Funktion, sie sind weder das Ergebnis intellektueller Reflexion, noch lösen sie beim Hörer Reflexion aus (Malinowski 1923/1936: 315). Aus diesem Grund sind sie für Malinowski (1923/1936: 315) nicht als ein Instrument der Übermittlung von Gedanken einzustufen, sondern als „mode of action, rather

38 Vergleiche dazu auch Senft (2009: 228): „[. . .] the terms ,phatic communion“ (and ,phatic communication') are generally used to refer to utterances that are said to have exclusively social, bonding functions like establishing and maintaining a friendly and harmonious atmosphere in interpersonal relations, especially during the opening and closing stages of social verbal - encounters“. 
than as a countersign of thought“ (1923/1936: 296, Herv. im Original). Über den puren Austausch von Äußerungen, auch wenn es eigentlich nichts zu sagen gibt, führt phatische Kommunion zu einer angenehmen Atmosphäre höflicher, sozialer Interaktion (Malinowski 1923/1936: 316), ${ }^{39}$ kann den Interagierenden den Einstieg in und den Ausstieg aus der Interaktion erleichtern (Laver 1975: 236) und zur Entschärfung, beispielsweise von als feindselig aufgefasstem Schweigen in Situationen, in denen Interaktion erwartet wird (Laver 1975: 220-221), beitragen.

Entgegen Lavers Standpunkt führen Coupland, Coupland und Robinson (1992: 213) an, dass phatische Kommunion nicht nur an den Rändern von Interaktionen auftritt (vgl. Laver 1975), sondern dass auf die Beziehung ausgerichtete und in einem phatischen Modus realisierte Interaktion ebenso in zielgerichteten Gesprächsphasen vorkommen kann. ${ }^{40}$ Dies unterstreicht auch Jaworski (2000: 113), der festhält, dass es unmöglich ist, „doing sociability“ gänzlich vom Austausch von Informationen zu separieren. Aus konversationsanalytischer Perspektive argumentieren Coupland, Coupland und Robinson (1992: 215, 217) dafür, dass von den Beteiligten im Zuge der Interaktion und mit Bezug auf ihre Beziehung gemeinsam ausgehandelt wird, ob eine Gesprächsphase als phatisch oder nicht phatisch behandelt wird - in Abhängigkeit von der sequenziellen Platzierung, dem jeweiligen Kontext und den jeweiligen interaktionalen Zielen.

Ähnlich wie phatische Kommunion dient auch Small Talk als beziehungsstiftendes Element in der Interaktion. Während Malinowski und Laver, wie oben dargestellt, von einer Zweckentbundenheit bei phatischer Kommunion ausgehen, zeigt neben Coupland, Coupland und Robinson (1992) auch Holmes (2005), dass Beziehungsmanagement mithilfe von Small Talk ebenfalls in institutionellen Zu-

39 Dies gilt laut Malinowski (1923/1936: 316) sowohl für „primitive“ als auch für „zivilisierte“ Interaktion. Bezüglich der Funktion von Wörtern zur Übermittlung von Gedanken führt Malinowski (1923/1936: 316) an: „It is only in certain very special uses among a civilized community and only in its highest uses that language is employed to frame and express thoughts. In poetic and literary production, language is made to embody human feelings and passions, to render in a subtle and convincing manner certain inner states and processes of mind. In works of science and philosophy, highly developed types of speech are used to control ideas and to make them common property of civilized mankind“.

40, ,[. . . [ we might see the fringes of interaction as a natural locus for relational goals to be foregrounded - that is, as sequences where a phatic design or ,frame' for talk (Goffman 1974) is particularly salient. On the other hand, by this account, phatic communion would cease to be associated uniquely with the fringes of encounters (Laver) or extended chatting (Malinowski, Cheepen) and we should expect to find instances where a relationally designed and perhaps phatic mode of talk surfaces whenever relational goals become salient - even within sequences of transactional, instrumental, or task-oriented talk“ (Coupland/Coupland/Robinson 1992: 213, Herv. im Original). 
sammenhängen betrieben wird. Hier kann Small Talk ein großes Spektrum an Funktionen erfüllen und eingesetzt werden, um beispielsweise Freundlichkeit zu kommunizieren, um Übereinstimmung zu etablieren sowie um Solidarität zwischen Angestellten aufrechtzuerhalten (Holmes 2005: 353). Small Talk kann sich auf typischerweise unkontroverse Themen wie das Wetter, ritualisierte Nachfragen zur Gesundheit, soziale Aktivitäten fernab der Arbeit, Sport, allgemeine Beschwerden (zum Beispiel über die Wirtschaft), positive Kommentare zum Aussehen einer Person oder die Arbeit beziehen (Holmes 2005: 353-354). Dabei müssen die Beteiligten auch die Fähigkeit besitzen, angemessen Small Talk zu betreiben, denn was im einen Kontext als angemessenes Small Talk-Thema gilt, kann in einem anderen Kontext als unangemessen aufgefasst werden (Holmes 2005: 354; vgl. auch Habscheid 2018). Auch eine falsche Einschätzung in Bezug auf gemeinsames Hintergrundwissen kann sich negativ auf Small Talk und damit verbunden auf die Beziehung zwischen den Beteiligten auswirken beziehungsweise dazu führen, dass Small Talk erst gar nicht zustande kommt (Holmes 2005: 354). In diesem Zusammenhang können auch soziale Faktoren, insbesondere die Art der Beziehung der Beteiligten, das Geschlecht sowie der hierarchische Status der Personen Small Talk beeinflussen (Holmes 2005: 354-355). Ein „sicheres“ Small Talk-Thema, auf das die Beteiligten zurückgreifen können, ist nach Holmes (2005: 356) das Thematisieren der Arbeit selbst, beispielsweise durch Verweise darauf, wie beschäftigt man gerade ist. Ähnlich wie es Laver (1975) für phatische Kommunion schildert, stellt Holmes (2005: 357) für Small Talk fest, dass dieser typischerweise an den „Rändern“ der Interaktion auftritt, wenn sich beispielsweise Angestellte am jeweiligen Tag zum ersten Mal begegnen oder zusammen am Arbeitsplatz eintreffen. Nichtsdestotrotz erscheinen Episoden des Small Talks auch an anderen Punkten in der Interaktion, beispielsweise um eine Brücke $\mathrm{zu}$ ausgedehnterem social talk zu schlagen (vgl. Holmes 2005: 360-361). Sowohl phatische Kommunion als auch Small Talk sind folglich nicht auf Interaktionsränder beschränkt, sondern können als Mittel zum Management der sozialen Beziehung zwischen den Interagierenden im gesamten Verlauf der Interaktion auftreten (vgl. Habscheid 2018).

In der funktionalen Pragmatik führen Ehlich und Rehbein (1980: 343) mit Bezug zu institutioneller Kommunikation (siehe Kapitel 2.3.2) das Konzept des homileïschen Diskurses ein, „um auf nicht-institutionelle Funktionen menschlicher Rede“ in institutionellen Zusammenhängen zu verweisen (Rehbein 2012: 85). ${ }^{41} \mathrm{Da}$ hinter steht die Beobachtung, dass beim Zusammentreffen von Menschen nicht

41 Vergleiche Ehlich und Rehbein (1980: 343): „Es gibt einen Bereich sprachlichen Handelns, der einerseits aufgrund seiner festen Organisation gewisse Gemeinsamkeiten mit dem Sprechen in Institutionen aufweist, andererseits dem institutionsspezifischen Handeln entgegengesetzt zu sein scheint. [. . .] Untersucht man Sprache in Institutionen, so wird man immer 
immer zielgerichtetes Handeln im Vordergrund steht, sondern auch lediglich das „Miteinander-Reden“, die „gesellschaftlich[e] Reproduktion“, den Zweck des Gesprächs darstellen kann (Rehbein 2012: 85). Den homileïschen Diskurs fasst Rehbein (2012: 89) als „de[n] gesellschaftliche[n] Ort, an dem sprachlich verhandelt wird, was in der Welt ,nicht aufgeht““. Pausengespräche stellen nach Rehbein (2012: 88, Herv. im Original; vgl. auch Ehlich/Rehbein 1980: 343), „,wenngleich oft nur in Ansätzen“, einen gesellschaftlichen Ort des homileïschen Diskurses dar, in dessen Rahmen den an ihm beteiligten Personen „eine psychische Erholung von der permanent geforderten, zielgerichteten Aufmerksamkeit am Arbeitsplatz zuteil“ wird. Neben dieser entlastenden Funktion kommt dem homileïschen Diskurs auch eine entschärfende Funktion, beispielsweise bei Streitigkeiten, zu (vgl. Rehbein 2012: 102).

Small Talk und phatische Kommunikation können durch ihr beziehungsstiftendes und -erhaltendes Potenzial soziale Vorraussetzungen beziehungsweise Rahmenbedingungen schaffen (vgl. Habscheid 2018), die es den Beteiligten erleichtern, eine potenziell heikle, gesichtsbedrohende sowie durch einen hohen sozialen Distinktionswert (vgl. Müller/Kluwe 2012: 5) gekennzeichnete Kommunikation über Kunst zu betreiben und ihnen zudem Ressourcen bieten, durch die Wahl bestimmter Themen aus dieser teils belastenden, fokussierten Interaktion auszusteigen (siehe Kapitel 8).

\subsection{Kontext und Situation}

Das Pausengespräch als institutionell vorgesehener Bestandteil der zeitlich und räumlich begrenzten sozialen Veranstaltung des Theaterbesuchs weist einerseits spezifische Ausprägungen und Abläufe wie beispielsweise das Verlassen des Theatersaals, das Versorgen mit Getränken oder die Rückkehr in den Theatersaal auf. Andererseits umfasst es auch bestimmte kommunikative Aktivitäten, unter anderem den kommunikativen Austausch über das Stück oder private Themen sowie damit verbunden den kommunikativen Abgleich von Erlebnissen und Wahrnehmungen. Im Vollzug dieser Aktivitäten können Small Talk und phatische Kommunikation - wie im vorangehenden Kapitel aufgezeigt - die Beteiligten bei der Aufgabe, das Rezipierte zu ver- und bearbeiten, unterstützen. Um beschreiben zu können, inwiefern der Kontext der geselligen Interaktion sowie die situativen Spezifika der in dieser Arbeit analysierten Pausengesprä-

wieder neben den auf die institutionsspezifischen Zwecke bezogenen Handlungen auf solche scheinbar für die Institution dysfunktionale Kommunikation stoßen [. . . ]“. 
che den Beteiligten als Ressource zur Unterstützung der Bewältigung der Aufgabe der Kunskommunikation dienen können, wird zunächst angeführt, wie die Begriffe Kontext und Situation aus ethnomethodologischer und gesprächsanalytischer Perspektive bestimmt sind.

Nach Schegloff weisen soziale Aktivitäten stets „einen kontextuell gebundenen Charakter“ (Bergmann 2001: 921) auf, indem Äußerungen und Handlungen der an der Interaktion Beteiligten sich „reflexiv auf den Kontext“ beziehen, in den sie eingebettet sind. Auch Bergmann (2001: 921) hält fest, dass die Äußerungen der Beteiligten immer wieder auf die Situation sowie den Kontext verweisen, in dem sie realisiert werden. Dies bedeutet, dass die Beteiligten immer auch Kontext und Situation ihrer Gespräche in den Blick nehmen müssen, um „Gehalt und Sinn einer Äusserung [sic!]“ nachvollziehen zu können (Bergmann 2001: 921).

Schegloff (1992: 195) differenziert zwischen einem externen und einem intrainteraktionalen Kontext: Der intra-interaktionale Kontext wird durch die Strukturen des vorangegangenen Gesprächs und das Betragen der Beteiligten hergestellt (vgl. Sidnell 2010: 245). Dieser Kontext umfasst laut Sidnell (2010: 245) die jeweilige Art und Weise der Interaktion, in der sich die Beteiligten als involviert verstehen, zum Beispiel eine wissenschaftliche Diskussion, ein belangloser Streit, ein vertrauliches Geplauder usw. Van Dijk (2007: 286) beschreibt diese Perspektive auf Kontext als die unmittelbare Situation ,of actual discourse production and understanding“, wie sie in Face-to-face-Interaktionen vorliegt, in denen die Beteiligten ko-präsent sind. Schegloff (1992: 195) charakterisiert diesen Kontext-Typ als „the sorts of sequences of talk or courses of conduct in which particular events may occur (stories, request sequences, etc.), the capacity in which participants act relative to the episode in progress (e. g. as initiator of a conversation or topic, or its recipient), etc.“. Der neben dem intra-interaktionalen Kontext von Schegloff (1992: 195) genannte externe Kontext umfasst größere Aspekte, beispielsweise der sozialen Stratifikation oder des institutionellen Zusammenhangs (vgl. auch Sidnell 2010: 245). Es geht dabei um eine weiter gefasste historische, gesellschaftliche oder kulturelle Situation, die nicht nur auf die aktuell Beteiligten, sondern auch auf Gruppen, Gemeinschaften oder Institutionen bezogen ist (van Dijk 2007: 286). Sie ist nicht auf die zeitlichen und örtlichen Gegebenheiten einer aktuellen Äußerung begrenzt, sondern umfasst eine weitere Zeitspanne und einen größeren Ort (van Dijk 2007: 286).

Aus konversationsanalytischer Perspektive wird bei der Bestimmung des jeweiligen Kontexts der Blick darauf gerichtet, wie die Interagierenden selbst Aspekte dieses Kontexts, in dem ihre Gespräche stattfinden, durch Verweise auf eben jene Aspekte relevant setzen (vgl. Sidnell 2010: 246). Die Frage ist also, inwieweit die Tatsache, dass das Gespräch in einem bestimmten Setting stattfindet, Konsequenzen für die Form, den Verlauf, den Inhalt oder den Interaktionscharakter 
des jeweiligen Gesprächs hat (Schegloff 1991: 53, zitiert nach Sidnell 2010: 246). Anders perspektiviert - so Schegloff (1992: 197) - scheint es angemessener, wenn nicht sogar eher der Fall zu sein, dass das Sprechen oder Handeln der Beteiligten einen bestimmten Kontext „aufruft“, als dass der Kontext das Sprechen und Handeln beeinflusst.

Auch Sidnell (2010: 247) konzeptioniert Kontext mit Bezug auf Heritage (1984) als dialektisches Modell. Am Beispiel von Gerichtsverhandlungen hält Sidnell (2010: 249) fest, dass sich die Beteiligten stetig am institutionellen Kontext, in dem sie agieren, orientieren. „This orientation shapes the ways in which the participants speak and thus they can be seen to talk the context into being.“ (Sidnell 2010: 249) Diese von Sidnell angeführte Orientierung trägt des Weiteren zur Gestaltung des gesamten Verlaufs des Gesprächs bei:

Indeed, it is not just that one participant is invoking or imposing the institutional context but, rather, that all the participants are co-constructing it. It is the participants' common and for the most part unquestioning engagement in this or that kind of sequence that provides for the felt presence of the institutional context.

(Sidnell 2010: 249, Herv. im Original)

Nach Sidnell wird der (institutionelle) Kontext, in dem die Beteiligten handeln, erst durch die Art und Weise der Realisierung der Handlungen erkennbar. Es geht aus konversationsanalytischer Perspektive bei der Analyse sprachlicher Interaktion darum, herauszustellen, wie die Interagierenden selbst Aspekte dieses Kontexts, in dem ihre Gespräche stattfinden, relevant setzen (vgl. Sidnell 2010: 246). Kontext ist folglich dynamisch zu denken, denn

every action is simultaneously context shaped (in that the framework of action from which it emerges provides primary organization for its production and interpretation) and context renewing (in that it now helps constitute the frame of relevance that will shape subsequent action).

(Goodwin/Heritage 1990: 289, Herv. im Original)

Die dynamische und reflexive Sichtweise bedeutet, dass das sprachliche Handeln der Beteiligten zum einen in einem bestimmten Kontext situiert, auf ihn zugeschnitten und so durch diesen geformt und zum anderen kontexterneuernd ist, indem die Beteiligten den Kontext erhalten, modifizieren, aktualisieren oder transformieren (vgl. Goodwin/Heritage 1990: 286-287).

Dass der Kontext von den Beteiligten interaktiv hervorgebracht wird, gilt ebenso für die Situierung von Gesprächen: Auch die jeweilige Gesprächssituation ist keine statische, die Gespräche umhüllende Größe, sondern die örtlichen und räumlichen Gegebenheiten sind „Bestandteile der kommunikativen (und über Kommunikation hinausreichenden) und zugleich soziokognitiven Praktiken, deren sinnhafte, individuell zugerechnete Einheiten ,Handlungen' und ihre kulturellen Verstehenshintergründe sind“ (Habscheid/Linz 2018: 1). Die in der vorliegenden Arbeit untersuchten Pausengespräche ermöglichen es den Beteiligten, 
sich in einem geselligen Rahmen, wenn auch in einer räumlich und zeitlich begrenzten Situation (siehe Kapitel 2.3.2), über das im Theaterstück Rezipierte auszutauschen. Mit ihren Kunstkommunikation relevant setzenden Äußerungen kommunizieren die Beteiligten nicht nur ihre Haltungen und nehmen Positionierungen vor, sondern es geht auch um die Demonstration von Wissen, das nach Müller und Kluwe (2012: 5) im Kontext der Kommunikation über Kunst immer auch ein exklusives Wissen ist und als soziales Distinktionsmerkmal dienen kann. Die Exklusivität dieser Art der Kommunikation, in der es Schwanitz (1999: 394) zufolge auch darum geht, „mit kultivierten Leuten mitzuhalten, ohne unangenehm aufzufallen“, ist allerdings nur nach außen gerichtet abgrenzend, während sich die Zuschauergruppen nach innen durch einen kooperativen Charakter auszeichnen (vgl. Habscheid 2018), in dem es um das Miteinander, also um Vergemeinschaftung, und nicht um ein Gegeneinander geht. Im prestigeträchtigen Kontext der Kunstkommunikation, also auch in den Pausengesprächen, ist wenn man mit Steinig (2016: 69) davon ausgeht, dass bildungssprachliches Handeln als Mittel für möglichen Prestigegewinn dienen kann - mit eben solchem sprachlichen Handeln zu rechnen (vgl. auch Habscheid 2018). Bildungssprachliches Handeln dient ebenso der Selbstdarstellung und regt laut Steinig (2016: 71) auch „Bildungsprozesse bei den Rezipienten an, die sich auf dieses Sprachverhalten einlassen“. Dies ist ein wichtiger Punkt für die in dieser Arbeit analysierten Bewertungsinteraktionen, in denen es schließlich auch darum geht, Wissen und Kompetenz zu zeigen und so andere Beteiligte möglicherweise von seinem Urteil zu überzeugen. Zu klären bleibt im empirischen Teil dieser Arbeit, inwiefern sich die Beteiligten auf bildungssprachliches Handeln beziehungsweise das entsprechende Sprachverhalten einlassen, fehlendes Wissen „durch geschickte Themenwechsel“ überspielen und über eine „flexibel einsetzbare Smalltalk-Kompetenz“ verfügen (Steinig 2016: 71; siehe auch Kapitel 4.1).

Für Themenwechsel von der Kunstkommunikation hin zu Small Talk-Themen bieten in der sozialen Situation des Theaterbesuchs örtliche Gegebenheiten Material (zum Beispiel die Spielstätte mit ihren Räumlichkeiten und Außenanlagen sowie in der semi-öffentlichen Situation anwesende Dritte), um mit den vielleicht durch andere im Rahmen der Kunstkommunikation über das Theaterstück aufgedeckten Wissenslücken umzugehen. Den geselligen und inkludierenden Charakter in ihren Gesprächen heben die Beteiligten unter anderem durch verschiedene Formen der Bezugnahmen hervor. So dient im folgenden Ausschnitt Emma und Monika ein Objekt im Außenbereich des Theaters als Ressource für eine scherzhafte Interaktionssequenz: 


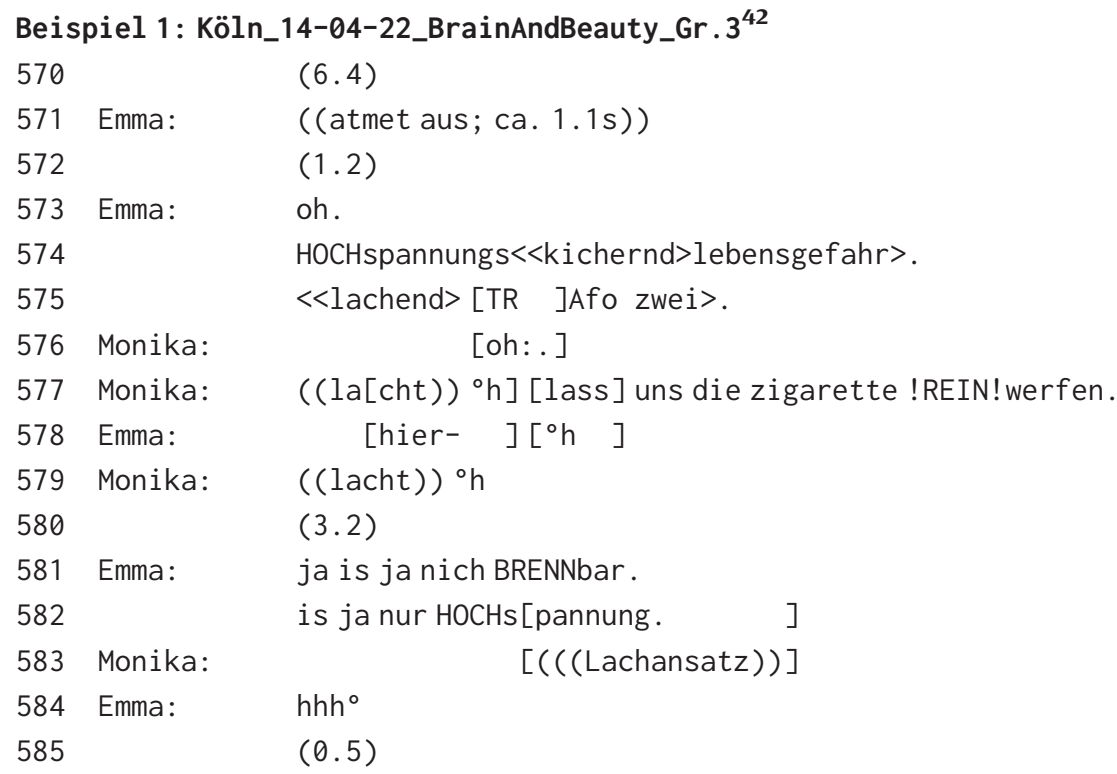

Auch Bezugnahmen auf beziehungsweise Lästereien über andere anwesende Personen werden von den Beteiligten als Ressource zur Vergemeinschaftung genutzt. So nimmt im folgenden Ausschnitt Hildegard zum Beispiel das Wiedererkennen einer anderen im Theaterfoyer anwesenden Person zum Anlass, um den anderen Mitgliedern ihrer Gruppe, Kristina und Irmgard, über eine kurze private Episode zu berichten:

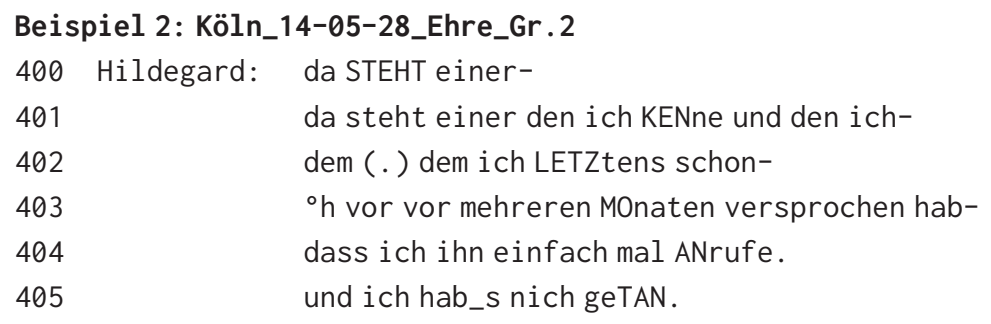

42 Die im Rahmen des DFG-Projeks (siehe Kapitel 1 und 6) vorgenommene und in dieser Arbeit übernommene Bezeichnung der Beispiele setzt sich zusammen aus dem Aufnahmeort, dem Aufnahmedatum, dem Titel des Theaterstücks sowie der Nummer der Teilnehmergruppe. Aufgrund unterschiedlicher Überarbeitungsstufen können sich die Transkripte in Teilen von den entsprechenden Transkripten in früheren aus dem Projekt hervorgegangenen Publikationen unterscheiden. 
406

407 Irmgard:

EINfallen?

408 Hildegard: [(ich) ich KANN-]

409 Irmgard: [wenn er dich S ]IEHT?

410 Hildegard: jetzt kann ich ihm doch nich schon WIEder sagen411

412 Kristina:

413 Irmgard: [(beRUFlich) oder was-

414 Kristina: [und wenn du dann nicht ANrufst?]

415 Hildegard: [doo:f. ]

416 Irmgard: [beRUFlich.]

417 Hildegard: ${ }^{\circ}$ hei: $n$.

418 Irmgard: [nein. ]

419 Hildegard: [eigentlich NICH so.]=ja: (.) im grunde is_es n beruflicher konTAKT?=Aber öhm: .

420

421 ${ }^{\circ}$ hh (. ) wir MÖgen uns so irgendwie? aber eigentlich weiß_ich gar nich was ich mit dem REden soll.

422 Irmgard: $\quad((\text { lacht }))^{\circ} \mathrm{h}$

423 Kristina: (oh) der HERR da?

424 Hildegard: nein ich sag jetzt !NICH! wer das is: .

$425 \quad(0.4)$

426 Hildegard: ich ZEIG da jetzt nIch drauf.

427 ich versuch ihn zu ignoRIEren.

428 Irmgard: ((lacht))

429 Hildegard: nich HINzugucken.

430

$(0.3)$

431 Kristina: ${ }^{\circ} \mathrm{hh}$ naJA.

432 Irmgard: also wenn er auf uns ZUkommt-

433

434

435 Hildegard: und dich freudig beGRÜSSTdann is die [sache geLÖST.

436 Irmgard:

437 Hildegard: [ich muss immer] auf IHN zukommen.

438 Irmgard: ((lacht))

439 Hildegard: er is_n ganz SCHÜCHterner.

440 Irmgard: $[(($ lacht $))]$

441 Kristina: $[(($ lacht $))]$

442 Hildegard: $[((\text { lacht }))]^{\circ} \mathrm{h}(($ seuftzt $))(.){ }^{\circ} \mathrm{h}$ 
In Beispiel 3 unterhalten sich Dina und ihre Mutter Susanne über die Haare einer ihnen unbekannten, im Theaterfoyer anwesenden Zuschauerin:

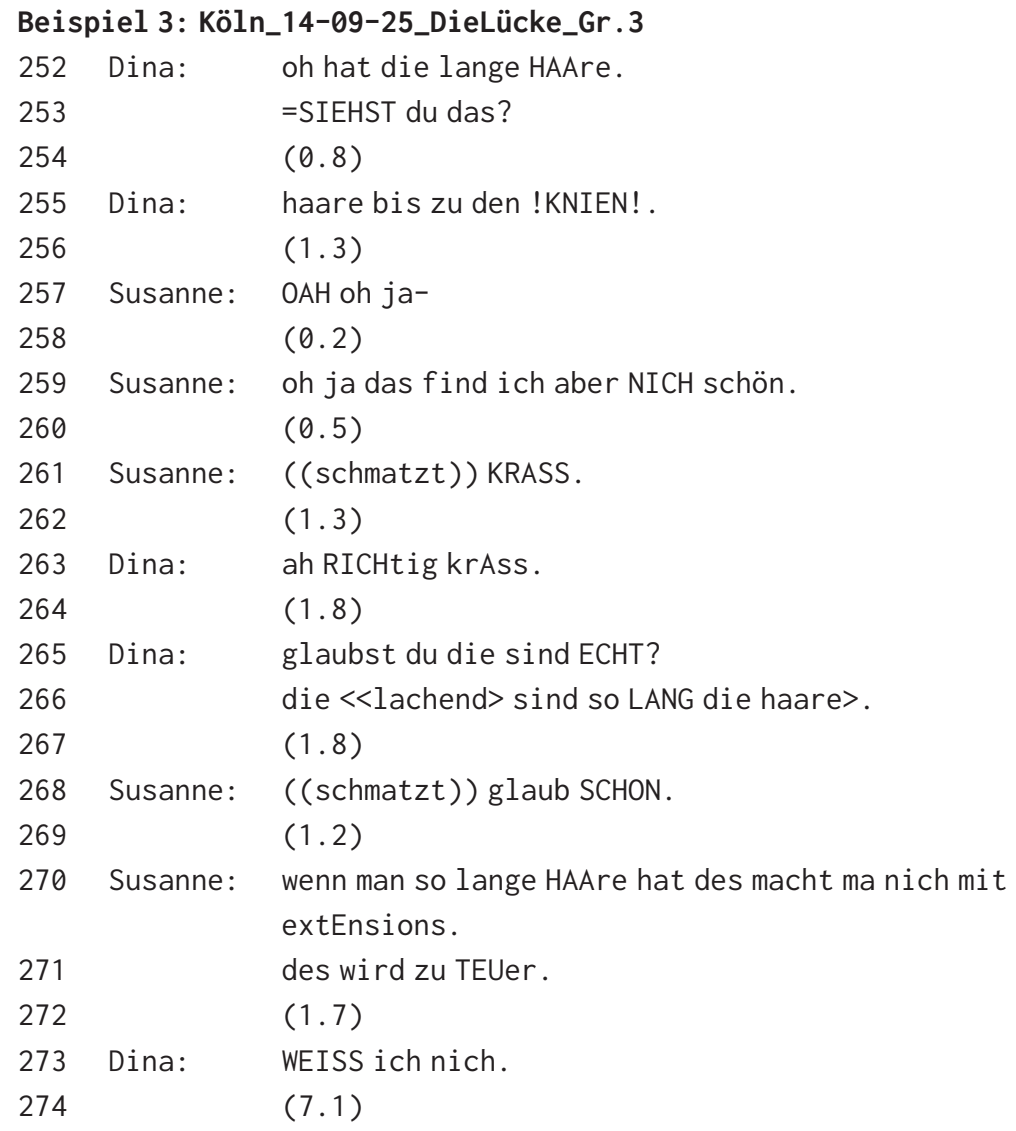

Wenig später nehmen die beiden auf einige der im Theaterpublikum vertretenen Personen Bezug und schreiben diesen dann ihrer Ansicht nach typische Theaterbesucher-Kategorien $\mathrm{zu}$ :

Beispiel 4: Köln_14-09-25_DieLücke_Gr.3

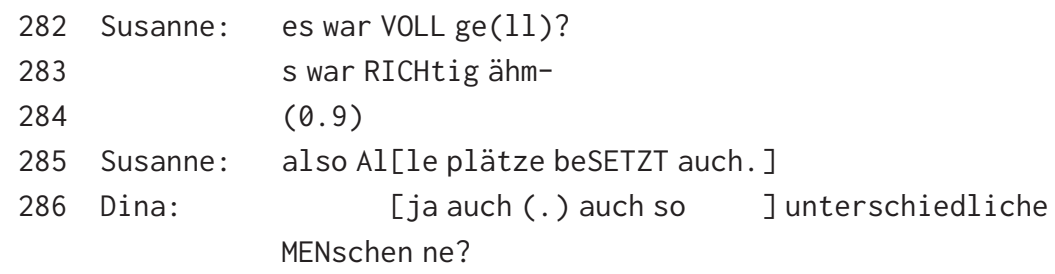




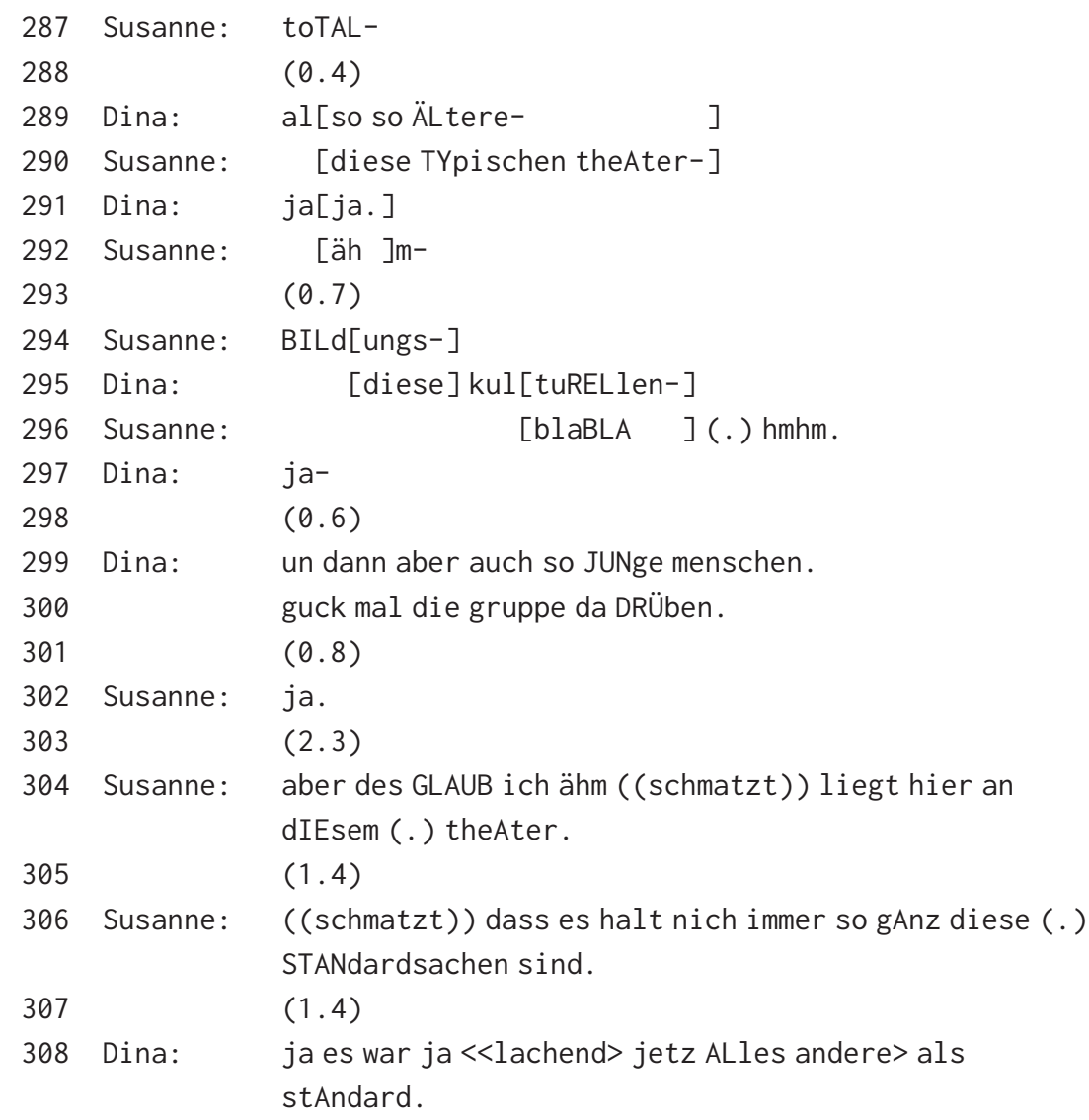

Themenwechsel vom Theaterstück und damit der Kommunikation über (performative) Kunst hin zu Themen, die in den Bereich der phatischen Kommunikation (siehe Kapitel 4.1) fallen, können - neben „primär physische[n] Praktiken“ (Habscheid/Linz 2018) wie beispielsweise dem Versorgen mit Getränken oder einem Spaziergang durch die Außenanlagen der Spielstätte - die Beteiligten dabei unterstützen, sich von der teilweise mit Anstrengung verbundenen Rezeption des Theaterstücks zu entlasten (vgl. Habscheid/Linz 2018).

Neben den oben erwähnten Bezugnahmen auf örtliche Gegebenheiten setzen die Beteiligten in ihren Gesprächen auch den zeitlichen Rahmen der Veranstaltung beziehungsweise des Pausengesprächs relevant. Dieser durch die Institution vorgegebene, zeitlich festgelegte Rahmen wird für die Theaterbesucher durch einen äußeren Rahmen (Beginn und Ende der Theatervorstellung) und einen inneren Rahmen (Beginn und Ende der Pause zwischen den Teilen des Theaterstücks) wahrnehmbar (vgl. Linz 2018). Während das Ausschalten des 
Lichts im Theatersaal sowie das Öffnen eines gegebenenfalls vorhandenen Vorhangs als äußerer Rahmen den Zuschauer erkennen lassen, dass „die Welt [. . .] nun auf der Bühne [stattfindet]“ (Hettlage 1991: 129), wird der innere Rahmen für die Theaterbesucher durch ein akustisches Signal, von Hettlage (1991: 129) auch als „raum-zeitliches Grenzzeichen“ bezeichnet, zum Ein- und Ausleuten der Pause wahrnehmbar (vgl. auch Linz 2018). Das akustische Signal zur zeitlichen Eingrenzung der Pause bildet gleichermaßen auch die „Anfangs- und Schlußklamme[r]“" (Hettlage 1991: 130, mit Bezug zu Goffmans Rahmenanalyse) des Pausengesprächs im Theater(foyer) und rahmt dieses als eine „Episode“ (Hettlage 1991: 129) der sozialen Veranstaltung „Theaterbesuch“. Solche „Anfangs- und Schlußklammern“ dienen, so Hettlage (1991: 129), der Verzahnung von „Ereignisse[n] mit der Umwelt“ sowie der Unterstreichung eines „,Innenraums“ gegenüber einer äußeren Wirklichkeit“" (vgl. auch Habscheid/Linz 2018; Linz/Hrncal/ Schlinkmann 2016: 526; Hrncal/Gerwinski 2015). Das Ende, mit Goffmans Worten die Schlussklammer der Theaterpause, ist für die Beteiligten allerdings in einigen Fällen nicht exakt vorhersehbar (vgl. Habscheid/Linz 2018), was oftmals bei der weiteren Planung des Pausengesprächs bedacht werden muss und von ihnen in ihren Gesprächen auch relevant gesetzt wird. Die von institutioneller Seite des Theaters zeitlich restringierte Pause und die Auswirkung dieser zeitlichen Einschränkung auf die Planung der Ausgestaltung der Pause werden in den Beispielen 5 und 6 deutlich.

So vergewissert sich Irmgard im folgenden Ausschnitt bei Cornelia nach der Länge der Pause, die ihr scheinbar als Entscheidungsfolie dafür dient, ob das Pausengespräch im Innen- oder Außenbereich des Theaters fortgeführt wird:

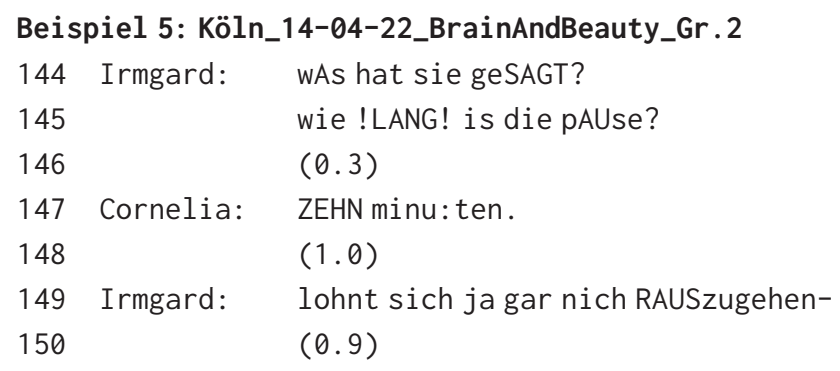

Die oben bereits angesprochene, zum Teil nicht vorhersehbare Pausenlänge zeigt sich in Beispiel 6 sowohl in der von Monika als auch von Emma jeweils mit dem Unsicherheitsmarker „glaub ich“ gerahmten Äußerung: 
Beispiel 6: Köln_14-04-22_BrainAndBeauty_Gr.3

\begin{tabular}{|c|c|c|}
\hline 198 & Emma: & {$[(($ atmet aus; ca. $1.1 \mathrm{~s}))]$} \\
\hline 199 & Monika: & [glaub ich RAUCH jetz lieber] nich so lang? \\
\hline 200 & & $(1.0)$ \\
\hline 201 & Emma: & wieSO; \\
\hline 202 & & $(0.8)$ \\
\hline 203 & Emma: & wir ham glaub ich genug ZEIT. \\
\hline 204 & & ${ }^{\circ} \mathrm{hhh}$ \\
\hline 205 & & $(2.4)$ \\
\hline
\end{tabular}

Insgesamt wird an den beiden angeführten Beispielen deutlich, dass die Pausenlänge als zeitliche Orientierung für die Beteiligten bei der Planung ihrer Gespräche eine wichtige Rolle spielt (siehe auch Kapitel 8.4.1).

\subsection{Zwischenfazit}

Die vorangehende Betrachtung von phatischer Kommunion, Small Talk und homileïschem Diskurs hat gezeigt, dass die drei Konzepte teilweise Gemeinsamkeiten aufweisen, andererseits aber auch zu differenzieren sind. Während es Ehlich und Rehbein (1980) bei ihrem Konzept des homileïschen Diskurses primär darum geht, auf Gesprächsabschnitte zu verweisen, die der Entlastung der Beteiligten von mit ihrem Arbeitsalltag verbundenen Aufgaben dienen und als nicht zielgerichtete Kommunikation zu beschreiben sind, verweist der Terminus ,phatische Kommunion' nach Malinowski (1923/1936) auf die gesprächsetablierende und -erhaltende Funktion von Kommunikation im Allgemeinen, die dazu dient, in einem bestimmten Modus soziale Beziehungen sowie eine angenehme Gesprächsatmosphäre herzustellen, aufrechtzuerhalten und Stille zu vermeiden. Als typische „Orte“ phatischer Kommunion nennen Malinowski (1923/1936) und auch Laver (1975) „Gesprächsränder“, das heißt Einstiege in sowie Ausstiege aus der Interaktion. Small Talk schließt bestimmte Themenbereiche ein, tritt als Bestandteil der beiden erstgenannten Phänomene auf und kann im Gespräch verschiedene Funktionen übernehmen. Es wird insgesamt deutlich, dass sich Small Talk und phatische Kommunion nicht nur auf Interaktionseinstiege und -ausstiege beschränken, sondern auch mitten in der Interaktion, parallel oder ergänzend zu aktuell von den Beteiligten bearbeiteten (sprachlichen) Aktivitäten stattfinden können. Welches Small Talk-Thema angemessen ist und wie lange Small Talk aufrechterhalten werden kann und sollte, unterliegt kontextspezifischen und situationsgebundenen Voraussetzungen, die von den Beteiligten durch ihr sprachliches Handeln stetig erneuert beziehungsweise aktualisiert werden 
(vgl. Goodwin/Heritage 1990). Festzuhalten bleibt mit Blick auf die in der vorliegenden Arbeit analysierten Gespräche vor allem, dass Episoden phatischer Kommunion und Small Talk den Beteiligten als entlastende Ressource oder sogar zur Entschärfung heikler Situationen dienen können. Diese Funktion kann nicht nur bei der Demonstration von Wissen (als ein Bestandteil von doing being gebildet) oder dem Aufdecken von Nichtwissen (vgl. Steinig 2016) eine große Rolle spielen, sondern auch für das Management nichtübereinstimmender Bewertungen im Rahmen eines geselligen Ereignisses, bei dem es auch um Unterhaltung und Vergnügen geht. Material für gesellige Kommunikation und die Möglichkeit des Sich-Vergnügens liefern unter anderem örtliche Gegebenheiten. Im gemeinsamen Wahrnehmungsraum vorhandene Objekte und Personen können den Beteiligten als Gesprächsanlass dienen und ihnen den Einstieg in oder den Ausstieg aus Bewertungsinteraktionen erleichtern. Nach Habscheid (2018) ist davon auszugehen, dass Small Talk im Fokus der Interaktion stehen kann, wenn es darum geht, ,alle Beteiligten in eine (gesellige) soziale Aktivität einzubinden“. Es kann aber auch zu Mischformen von Small Talk und institutioneller Kommunikation kommen oder Small Talk tritt in den Hintergrund ,während die sachbezogene Kommunikation im Vordergrund steht“" (Habscheid 2018).

Für die in der vorliegenden Arbeit untersuchten Gespräche bedeutet die in Kapitel 4.2 skizzierte Konzeptionierung von Kontext, dass die an den Bewertungsinteraktionen Beteiligten ihre Äußerungen mit Blick auf den Kontext der Kunstkommunikation sowie der geselligen Interaktion und mit Blick auf die örtlichen und zeitlichen Gegebenheiten realisieren, diese so relevant setzen sowie aufrufen, und diese Kontexte und die Situation ihrer Gespräche durch ihre Äußerungen fortwährend erneuern. Die (musterhaften) Ausprägungen ihrer Äußerungen sind deshalb lediglich für die jeweilige Situation aktuell.

Nachdem in den vorangegangenen Kapiteln mit der Diskussion verschiedener Bewertungskonzepte und mit einem Blick auf Forschungsarbeiten zum Bewerten in der Interaktion aus einer konversationsanalytischen Perspektive sowie mit der Skizzierung der Rolle von Bewertungen im Kontext der Zuschauer- und der geselligen Kommunikation das theoretische Fundament für die im empirischen Teil dieser Arbeit vorgenommene Analyse gelegt wurde, wird im folgenden Kapitel die methodische Basis für die Untersuchung von Bewertungsinteraktionen in den vorliegenden Gesprächen erläutert. 